\title{
Vertex Equitable Labeling of Cyclic Snakes and Bistar Graphs
}

\author{
P. Jeyanthi ${ }^{{ }^{*}}$ and A. Maheswari ${ }^{2}$ \\ ${ }^{1}$ Research Centre, Department of Mathematics, Govindammal Aditanar College for Women, \\ Tiruchendur-628 215, Tamilnadu,India \\ ${ }^{2}$ Department of Mathematics, Kamaraj College of Engineering and Technology, Virudhunagar, \\ Tamilnadu, India
}

Received 21 May 2013, accepted in final revised form 18 December 2013

Abstract
Let $G$ be a graph with $p$ vertices and $q$ edges and let $A=\left\{0,1,2, \ldots,\left\lceil\frac{q}{2}\right\rceil\right\}$.

A vertex labeling $f: V(G) \rightarrow A$ induces an edge labeling $f^{*}$ defined by $f^{*}(u v)=f(u)+f(v)$ for all edges $u v$. For $a \in A$, let $v_{f}(a)$ be the number of vertices $v$ with $f(v)=a$. A graph $G$ is said to be vertex equitable if there exists a vertex labeling $f$ such that for all $a$ and $b$ in $A$, $\left|v_{f}(a)-v_{f}(b)\right| \leq 1$ and the induced edge labels are $1,2,3, \ldots, q$. In this paper, we establish vertex equitable labeling of square graph of $B_{n, n}$ and splitting graph of $B_{n, n}$ and $k C_{4}-$ snake $(k \geq 1)$ and the generalized $k C_{n}-$ snake is vertex equitable if $n \equiv 0(\bmod 4), n \geq 4$.

Keywords: Vertex equitable labeling; Vertex equitable graph.

○ 2014 JSR Publications. ISSN: 2070-0237 (Print); 2070-0245 (Online). All rights reserved. doi: http://dx.doi.org/10.3329/jsr.v6i1.15044 J. Sci. Res. 6 (1), 79-85 (2014)

\section{Introduction}

All graphs considered here are simple, finite, connected and undirected .We follow the basic notations and terminologies of graph theory as in [1]. A graph labeling is an assignment of integers to the vertices or edges or both, subject to certain conditions. There are several types of labeling. A detailed survey of graph labeling can be found in ref. [2].

The concept of vertex equitable labeling was introduced by Lourdusamy and Seenivasan [3]. Let $G$ be a graph with $p$ vertices and $q$ edges and $A=\left\{0,1,2, \ldots,\left\lceil\frac{q}{2}\right\rceil\right\}$. A graph $G$ is said to be vertex equitable if there exists a vertex labeling $f: V \rightarrow A$ that induces an edge labeling $f^{*}$ defined by $f^{*}(u v)=f(u)+f(v)$ for all edges $u v$ such that for all

\footnotetext{
*Corresponding author: jeyajeyanthi@rediffmail.com
} 
$a$ and $b$ in $A,\left|v_{f}(a)-v_{f}(b)\right| \leq 1$ and the induced edge labels are $1,2,3, \ldots, q$, where $v_{f}(a)$ be the number of vertices $v$ with $f(v)=a$ for $a \in A$. The vertex labeling $f$ is known as vertex equitable labeling. A graph $G$ is said to be a vertex equitable graph if it admits vertex equitable labeling. They proved that the graphs like path, bistar $B(n, n)$, combs, cycle $\mathrm{C}_{\mathrm{n}}$ if $n \equiv 0$ or $3(\bmod 4), K_{2, \mathrm{n}}, C_{3}{ }^{(\mathrm{t})}$ for $t \geq 2$, quadrilateral snake, $K_{2}+m K_{l}, K_{l, n} U$ $K_{l, n+k}$ iff $1 \leq k \leq 3$, ladder, arbitrary super division of any path and cycle $C_{n}$ with $n \equiv 0$ or 3 (mod4) are vertex equitable. Also they proved that the graphs $K_{l, n}$ if $n \geq 4$, any Eulerian graph with n edges where $n \equiv 1$ or $2(\bmod 4)$, the wheel $W_{n}$, the complete graph $K_{n}$ if $n$ $>3$ and triangular cactus with $q \equiv 0$ or 6 or $9(\bmod 12)$ are not vertex equitable. In addition, they proved that if $G$ is a graph with $p$ vertices and $q$ edges, $q$ is even and $p<$ $\left\lceil\frac{q}{2}\right\rceil+2$ then $G$ is not vertex equitable graph.

Jeyanthi and Maheswari [4-7] proved that $T_{\mathrm{p}}$-tree, $T \square \overline{K_{n}}$ where $T$ is a $T_{p}$-tree with even number of vertices, $T$ ô $P_{n}, T$ ô $2 P_{n}, T \hat{o} C_{n}(n \equiv 0,3(\bmod 4)), T \tilde{o} C_{n}(n \equiv 0,3$ $(\bmod 4))$, bistar $B(n, n+1)$, the caterpillar $\quad S\left(x_{1}, x_{2}, \ldots, x_{n}\right)$ and $C_{n} \square K_{1}, P_{n}{ }^{2}$ ,tadpoles, $C_{m} \oplus C_{n}$, armed crowns, $\left[P_{m} ; C_{n}{ }^{2}\right],\left\langle P_{m} \hat{o} \mathrm{~K}_{1, \mathrm{n}}\right\rangle$ and the graphs obtained by duplicating an arbitrary vertex and an arbitrary edge of a cycle $C_{n}$, total graph of $P_{n}$, splitting graph of $P_{n}$ and fusion of two edges of a cycle $C_{n}$ are vertex equitable graphs. In this paper we prove that $k C_{4}$-snakes for all $k \geq 1$ are vertex equitable graph and generalized $k \mathrm{C}_{\mathrm{n}}$-snake is vertex equitable if $n=0(\bmod 4), n \geq 4$. Also we prove that square graph of $B_{n, n}$ and splitting graph of $B_{n, n}$ are vertex equitable.

The following definitions are used in the present study.

Definition 1.1 A $k C_{n}$-snake is defined as a connected graph in which all the $k$-blocks are isomorphic to the cycle $C_{n}$ and the block-cut point graph is a path. Let $\mathrm{P}$ be the path of minimum length that contains all the cut vertices of a $k C_{n}$-snake. Barrientos [8] has proved that any $k C_{n}$-snake is represented by a string $\mathrm{s}_{1}, \mathrm{~s}_{2}, \ldots, \mathrm{s}_{k-2}$ of integers of length $k-2$ where the $i^{\text {th }}$ integer, $s_{i}$ on the string is the distance between $i^{\text {th }}$ and $(i+1)^{\text {th }}$ cut vertices on the path $P$ from one extreme and is taken from $S_{n}=\left\{1,2, \ldots,\left\lfloor\frac{n}{2}\right\rfloor\right\}$.The strings obtained for both extremes are assumed to be the same. Then there are at most $\left\lfloor\frac{n}{2}\right\rfloor^{k-2}$ non isomorphic $k C_{n}$-snakes. For example, the string of a $10 C_{4}$-snake is shown in Fig.1 is 2,2,1,2,1,1,2,1, , , .A $k C_{n}$-snake is said to be linear if each integer of its string is $\left\lfloor\frac{n}{2}\right\rfloor$. 


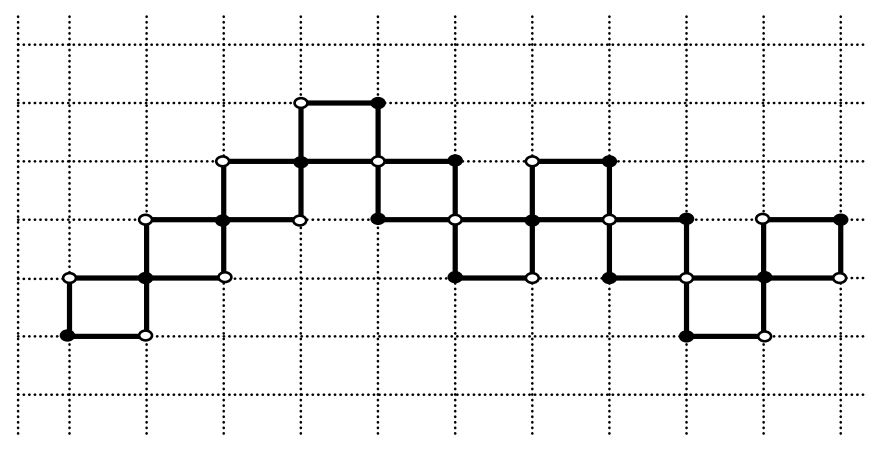

Fig. 1.

Definition 1.2 A generalized $k C_{n}$-snake is defined as a connected graph in which each block is isomorphic to a cycle $C_{n}$ for some $n$ and the block-cut point graph is a path. It is denoted by $C S\left(n_{1}, n_{2}, \ldots, n_{k}\right)$ where $B_{1}, B_{2}, \ldots, B_{k}$ are the consecutive blocks and $B_{i}$ is isomorphic to $C_{n_{i}}$. By applying the same methods used to obtain the strings of a $k C_{n^{-}}$ snake, we can show that any generalized $k C_{n}$-snake can also be represented by a string of integers $s_{1}, s_{2}, \ldots, s_{k-2}$ of length $k-2$ where $s_{i-1} \in S_{n_{i}}$.

Definition 1.3 The square of a graph $G$ denoted by $G^{2}$ has the same vertex set $V(G)$ and the two vertices are adjacent in $G^{2}$ if they are at a distance of 1 or 2 in $G$.

Definition 1.4 Let $G$ be a graph. For each point $v$ of a graph $G$, take a new point $v^{\prime}$ and join $v^{\prime}$ to the vertices of $G$ which are adjacent to $v$. The graph thus obtained is called the splitting graph of $\mathrm{G}$ and is denoted by $S^{\prime}(G)$.

\section{Main Results}

Theorem 2.1: The $k C_{4}-$ snake is a vertex equitable graph.

Proof: Let $\mathrm{G}$ be a $k C_{4}$-snake with $k$ blocks. As $\mathrm{G}$ is bipartite, let one partite set has black vertices and the other white vertices. Therefore, $\mathrm{G}$ can be embedded on a square grid as shown in Figure 1.

Consider the assignment of numbers to the vertices of $C_{4}$ as shown in Fig. 2 where $x$ is an even integer. Then the induced edge labels are $2 x+1,2 x+2,2 x+3,2 x+4$ four successive integers starting from $2 x+1$.

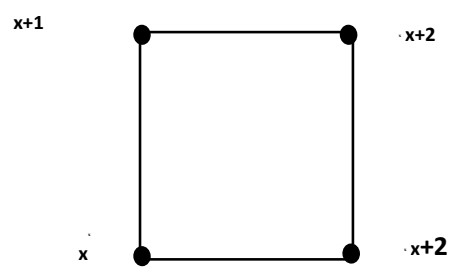

Fig. 2. 
The vertices of $G$ are labeled as follows: The black vertices in the diagonals, except the first vertex of each diagonal, ordered from left to right and inside the diagonals from bottom to top are assigned the numbers from an arithmetic progression of common difference and first term both equal to 2 .

The first vertex in the first diagonal is labeled with 0 and the first vertex in each of the remaining diagonals is labeled with the integer which is one more than the label assigned with the last vertex of the previous diagonal.

The white vertices in the diagonals, except the first vertex of each diagonal are ordered from left to right and inside the diagonals from top to bottom are assigned the numbers from an arithmetic progression of common difference 2 and first term 2.

The first vertex in the first diagonal is labeled with 1 and the first vertex in each of the remaining diagonals is labeled with the integer which is one more than the label assigned for the last vertex of the previous diagonal.

The labeling of each copy of $\mathrm{C}_{4}$ is the same as in Figure 2 and the black and white vertices are labeled respectively with integers from $0,1,2, \ldots, 2 k$. It can be easily verified that the set of induced edge labels defined by the sum of the labels on the end vertices is the set $1,2, \ldots, 4 k$ and $\left|v_{f}(a)-v_{f}(b)\right| \leq 1$ for all $a, b \in 0,1,2, \ldots, 2 k$. Then $G$ is a vertex equitable graph.

An example for the vertex equitable labeling of $10 C_{4}$-snake is shown in Fig. 3.

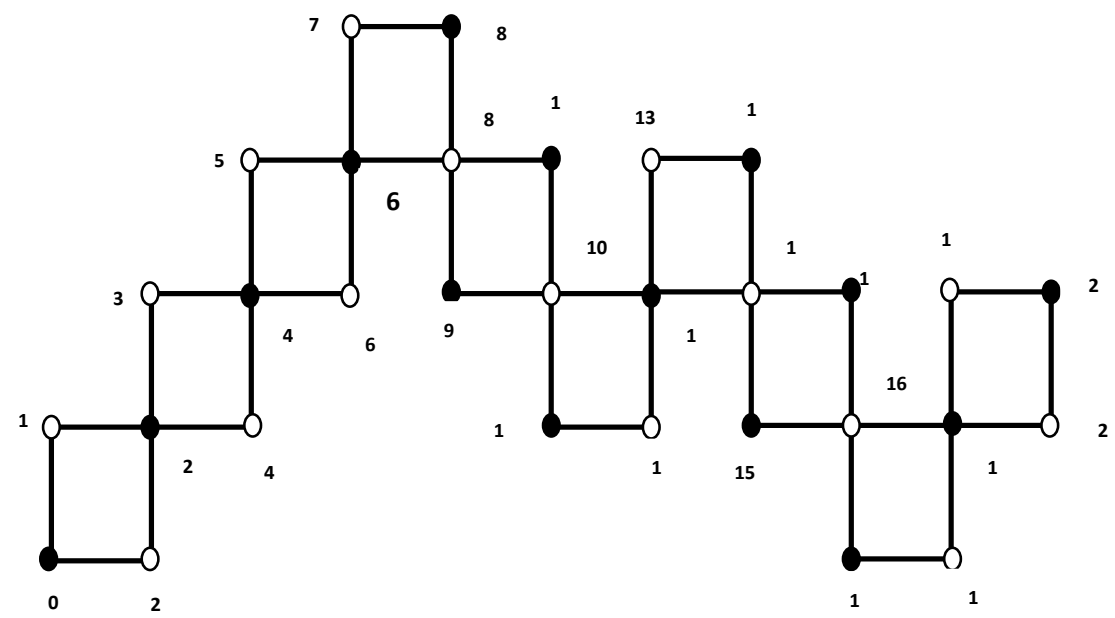

Fig. 3

Theorem 2.2: Let $G=\operatorname{CS}\left(n_{1}, n_{2}, \ldots, n_{\mathrm{k}}\right), n_{i} \equiv 0(\bmod 4), n_{i} \geq 4$, be a generalized $k \mathrm{C}_{\mathrm{n}}-$ snake with its strings $\mathrm{s}_{1}, \mathrm{~s}_{2}, \ldots, \mathrm{s}_{k-2}$ where $s_{i} \in 1,2,1 \leq i \leq k$. Then $\mathrm{G}$ is a vertex equitable graph. 
Proof: Let $B_{1}, B_{2}, \ldots, B_{k}$ be the consecutive blocks of $G$. Here each block $B_{i}$ is isomorphic to $C_{n i}$. Let $V\left(C_{n i}\right)=v_{i j}: 1 \leq j \leq n_{i}$ be the vertex set of the cycle $C_{n i}(1 \leq i \leq k)$. First we define a vertex labeling $f_{i}$ for the cycle $C_{n i}(1 \leq i \leq k)$, as $f_{i}: V\left(C_{n i}\right) \rightarrow$ $\left\{x, x+1, x+2, \ldots, x+\frac{n_{i}}{2}\right\}$ as follows with $x=0$ if $i=1$ and $x=\frac{n_{1}+n_{2}+\ldots+n_{i-1}}{2}$ if $i=2,3, \ldots, k . f\left(v_{i j}\right)= \begin{cases}x+\left\lfloor\frac{j}{2}\right\rfloor & \text { if } 1 \leq j \leq \frac{n_{i}}{2} \\ x+\left\lceil\frac{j}{2}\right\rceil & \text { if } \quad \frac{n_{i}}{2}+1 \leq j \leq n_{i} .\end{cases}$

Then the edge labels induced on the blocks $B_{i}(1 \leq i \leq k)$ are $\sum_{r=1}^{i-1} n_{r}+1, \sum_{r=1}^{i-1} n_{r}+2, \ldots$, $\sum_{r=1}^{i} n_{r}$. Therefore, the edge labels induced on $G$ are $1,2,3, \ldots, \sum_{r=1}^{k} n_{r}$. To get the vertex equitable labeling of $G$, identify the vertex $v_{21}$ of $C_{n 2}$ with either $v_{n 1}$ or $v_{n_{1}-1}$ of $C_{n 1}$ and identify the vertex $v_{i+11}$ of $C_{n_{i}+1}$ with $v_{i n_{i}}$ of $C_{n i}$ if $s_{i}=1$ or with $v_{i n_{i}-1}$ if $s_{i}=2$ for $2 \leq i \leq k$

An example for the vertex equitable labeling of $C S(8,4,12,8,16)$ is shown in Fig. 4.

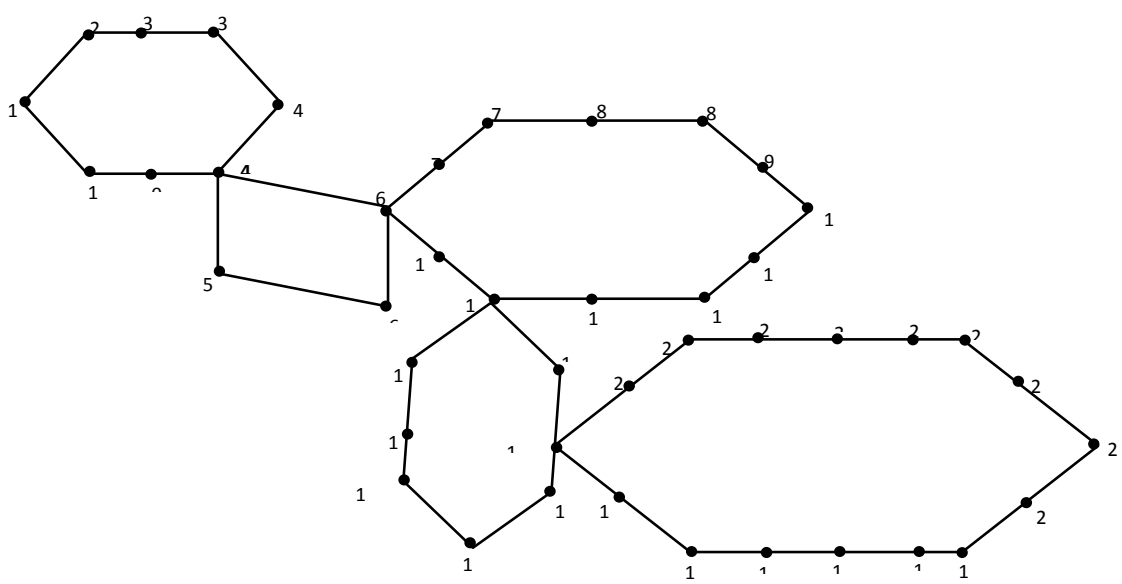

Fig. 4 
Theorem 2.3: The square graph $B_{n, n}^{2}$ is a vertex equitable graph.

Proof: Let $V\left(B_{n, n}\right)=u, v, u_{i}, v_{i}, 1 \leq i \leq n$ where $u_{i}, v_{i}$ are the pendant vertices adjacent to $u$ and $v$ respectively. Let $G=B_{n, n}^{2}$. Then $|V(G)|=2 n+2$ and $|E(G)|=4 n+1$. Let $A=\{0$, $\left.1,2, \ldots,\left\lceil\frac{4 n+1}{2}\right\rceil\right\}$. Define $f: V\left(B_{n, n}^{2}\right) \rightarrow A$ as follows: $f(u)=0, f(v)=\left\lceil\frac{4 n+1}{2}\right\rceil, f\left(u_{i}\right)=i, 1 \leq i$ $\leq n$ and $f\left(v_{i}\right)=n+i, 1 \leq i \leq n$. It can be verified that the induced edge labels of $B_{n, n}^{2}$ are 1 , $2, \ldots, 4 n+1$ and $\left|v_{f}(i)-v_{f}(j)\right| \leq 1$ for $i, j \in A$. Therefore, $B_{n, n}^{2}$ is a vertex equitable graph.

An example for the vertex equitable labeling of $B_{4,4}^{2}$ is given in Fig.5.

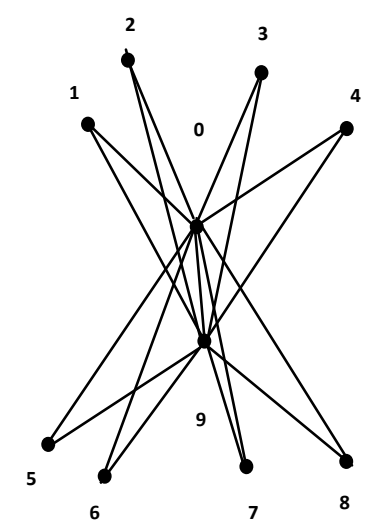

Fig. 5

Theorem 2.4: The splitting graph $S^{\prime}\left(B_{n, n}\right)$ is a vertex equitable graph.

Proof: Let $V\left(B_{n, n}\right)=u, v, u_{i}, v_{i}, 1 \leq i \leq n$ where $u_{i}, v_{i}$ are the pendant vertices adjacent to $u$ and $v$ respectively. Let $u^{\prime}, v^{\prime}, u_{i}^{\prime}, v_{i}^{\prime}$ be the vertices corresponding to $u, v, u_{i}, v_{i}$ ( $1 \leq i \leq n)$ in $S^{\prime}\left(B_{n, n}\right)$ and let $G=S^{\prime}\left(B_{n, n}\right)$. Then $|V(G)|=4(n+1)$ and $|E(G)|=6 n+3$. Let $A=\left\{0,1,2, \ldots,\left\lceil\frac{6 n+3}{2}\right\rceil\right\}$. Define $f: V\left(S^{\prime}{ }^{\prime}\left(B_{n, n}\right)\right) \rightarrow A$ as follows: $f(u)$ $=0, f(v)=\left\lceil\frac{6 n+3}{2}\right\rceil, f\left(u^{\prime}\right)=n+1, f\left(v^{\prime}\right)=2 n+1$. For $1 \leq i \leq n \quad f\left(u_{i}\right)=n+i \quad, f\left(u_{i}^{\prime}\right)=i, f\left(v_{i}\right)$ $=n+1+i$ and $f\left(v_{i}^{\prime}\right)=2 n+1+i$. It can be verified that the induced edge labels of $S^{\prime}\left(B_{n, n}\right)$ 
are $1,2, \ldots, 6 n+3$ and $\left|v_{f}(i)-v_{f}(j)\right| \leq 1$ for $i, j \in A$. Therefore, $S^{\prime}\left(B_{n, n}\right)$ is a vertex equitable graph.

An example for the vertex equitable labeling of $S^{\prime}\left(B_{4,4}\right)$ is given in Fig. 6.

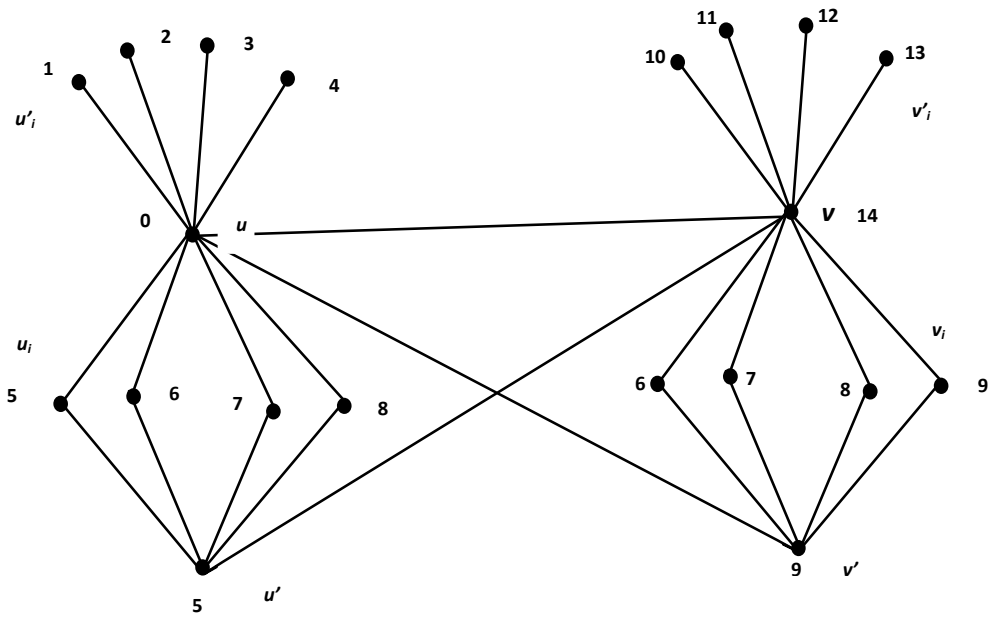

Fig. 6

\section{References}

1. F. Harary, Graph theory (Addison Wesley, Massachusetts, 1972). PMid:4261464.

2. J. A. Gallian, The Electronic J. Combinatorics DS6 (2012).

3. A. Lourdusamy and M.Seenivasan, J. Discrete Math. Sci. Cryptography 11 (6), (2008). http://dx.doi.org/10.1080/09720529.2008.10698401

4. P. Jeyanthi and A. Maheswari, Open J. Discrete Math. 2, 51 (2012).

5. P. Jeyanthi and A. Maheswari, Vertex equitable labeling of cycle and path related graphs, Utilitas Mathematica, to appear (2014).

6. P. Jeyanthi and A. Maheswari, J Algorit. Comput. 44 (1), 9 (2013).

7. P. Jeyanthi and A. Maheswari, Vertex equitable labeling of cycle related graphs-,Graph Theory Notes of Newyork, Preprint (June 2013).

8. C. Barrientos, Ars Combinatoria 60, 85 (2001). 